\title{
Erratum: A microscopic view on the Mott transition in chromium-doped $\mathrm{V}_{2} \mathrm{O}_{3}$
}

\author{
S. Lupi, L. Baldassarre, B. Mansart, A. Perucchi, A. Barinov, P. Dudin, E. Papalazarou, F. Rodolakis, J.-P. Rueff, \\ J.-P. Itié, S. Ravy, D. Nicoletti, P. Postorino, P. Hansmann, N. Parragh, A. Toschi, T. Saha-Dasgupta, \\ O. K. Andersen, G. Sangiovanni, K. Held \& M. Marsi
}

Nature Communications 1:105 doi: 10.1038/ncomms1109 (2010); Published 2 Nov 2010; Updated 17 Jan 2012.

In Figure 2 of this Article, panel labels $\mathbf{c}$ and $\mathbf{d}$ were inadvertently switched. A typographical error was also introduced in the last sentence of the legend, which should have read 'The scale bar in panel c represents $10 \mu \mathrm{m}$ '. The correct version of the figure and its legend appears below.
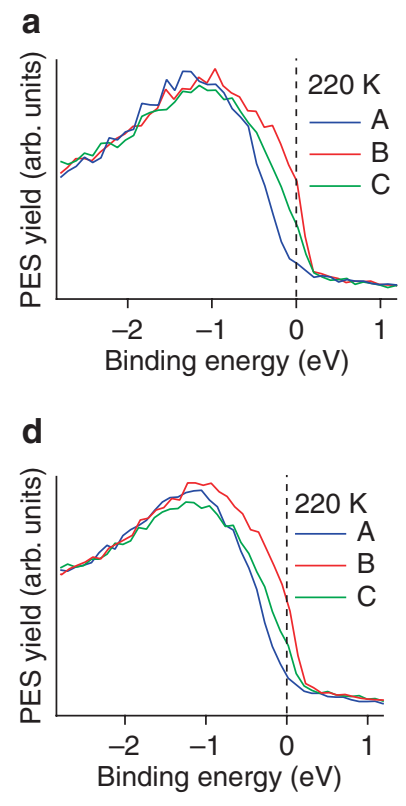
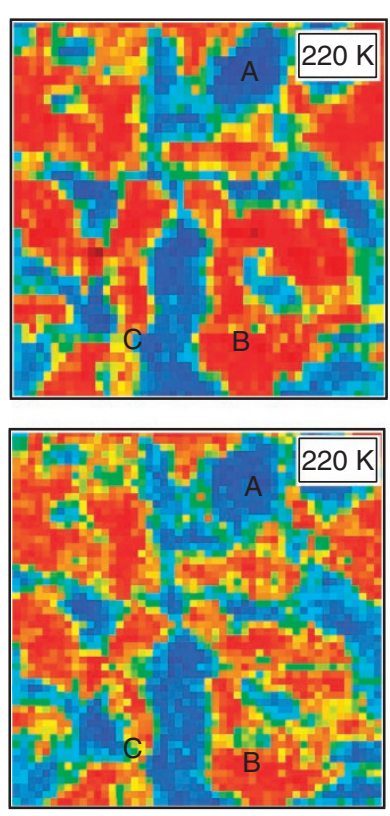
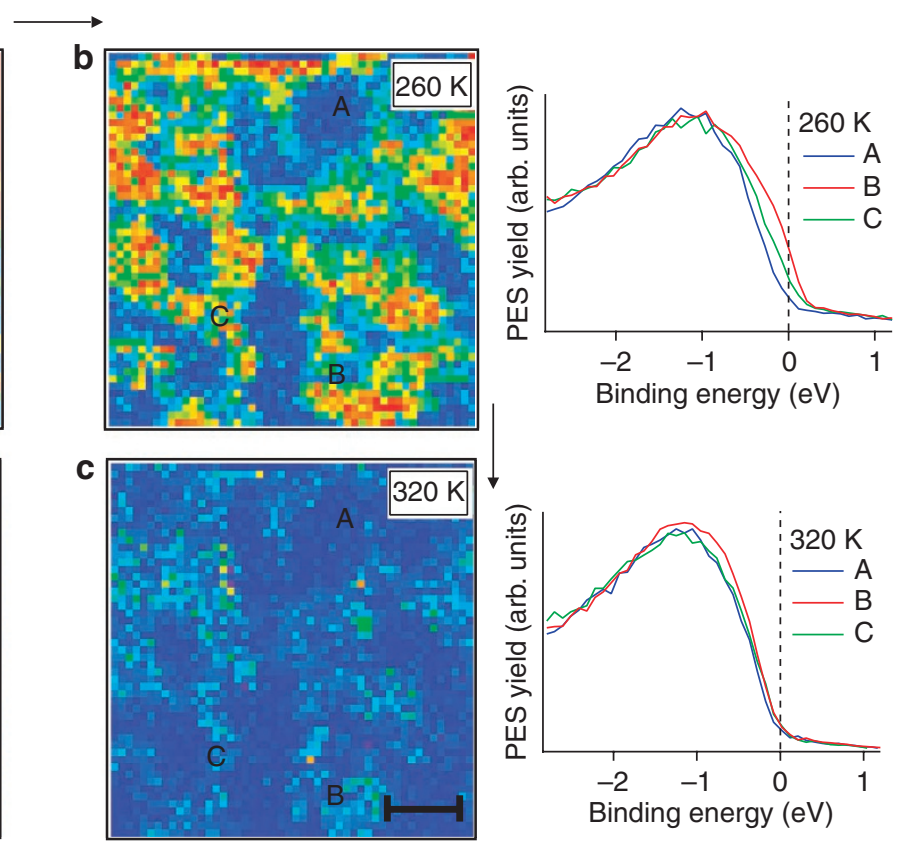

Figure 2 | Temperature-dependent photoemission microscopy measurements. Scanning photoemission microscopy images and spectra on $\left(\mathrm{V}_{0.989} \mathrm{Cr}_{0.011}\right)_{2} \mathrm{O}_{3}$, collected at $27 \mathrm{eV}$ photon energy and at different temperatures on a $50 \times 50 \mu \mathrm{m}$ sample area. The images are obtained with standard procedures to remove background and topography effects ${ }^{16}$. The pictorial contrast between metallic and insulating zones is obtained from the

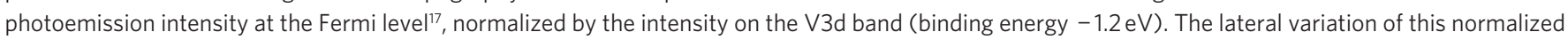

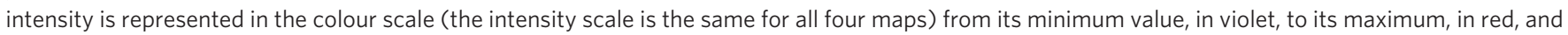
is therefore a direct visualization in real space of the metallicity of the system. Inhomogeneous properties are found within the PM phase at $T=220 \mathrm{~K}$ (a)

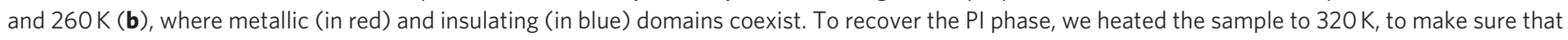
the hysteresis effects still present at $300 \mathrm{~K}$ (see Fig. 1) are overcome: at this temperature (c) a homogenous insulating state is obtained. After a whole thermal cycle (d) the metallic regions can be found in the same position and shape as in (a). The photoemission spectra (outer panels) from selected representative areas $(A, B, C)$ corroborate this interpretation. The scale bar in panel c represents $10 \mu \mathrm{m}$. 